\title{
Long-Term Effectiveness of Three Anti-CGRP Monoclonal Antibodies in Resistant Chronic Migraine Patients Based on the MIDAS score
}

\author{
Luigi Francesco lannone ${ }^{1,2} \cdot$ Davide Fattori $^{1} \cdot$ Silvia Benemei $^{2} \cdot$ Alberto Chiarugi $^{1,2} \cdot$ Pierangelo Geppetti $^{1,2}$. \\ Francesco De Cesaris ${ }^{2}$
}

Accepted: 22 December 2021 / Published online: 11 February 2022

(c) The Author(s) 2022

\begin{abstract}
Background Criteria, including clinical features and effective outcomes, for access and persistence of novel but costly treatments may vary between countries, thus affecting the health of patients. Monoclonal antibodies against the calcitonin gene-related peptide pathway (anti-CGRP mAbs) for migraine treatment are currently prescribed following strict criteria. Objective The aim was to assess the effectiveness and safety of three anti-CGRP mAbs (erenumab, galcanezumab, and fremanezumab) in consecutive resistant chronic migraine patients presenting at our Headache Center and the impact of criteria set by the Italian Medicines Agency to start and continue (achieving a $\geq 50 \%$ reduction in Migraine Disability Assessment [MIDAS] score) with treatment under the reimbursement program.

Methods A monocentric, prospective, cohort study was conducted, enrolling 203 severe (resistant to three or more preventive treatments) chronic migraine patients ( $84.7 \%$ with medication overuse) treated with erenumab (47.2\%), galcanezumab (36.5\%), or fremanezumab (16.3\%), with up to 12 months follow-up. Patients completed a headache diary that included monthly migraine days (MMDs), number of analgesics and days with analgesic use, and patient-reported outcome questionnaires (MIDAS, Headache Impact Test 6 [HIT-6] questionnaires, and the Patient Global Impression of Change [PGIC] scale). Moreover, percentages of patients showing $\geq 50 \%, \geq 75 \%$ and $100 \%$ reduction in MMDs (responder rates) were calculated at different follow-ups. A subgroup analysis was performed for patients with 12-month follow-up. Potential predictors of response were assessed at different follow-ups.

Results In the overall population, all three anti-CGRP mAbs were similarly effective and dropouts were $17.2 \%$. The percentage of patients with $\geq 50 \%$ reduction in MMDs ( $\min -\max 36.4-56.8 \%$ ) and in monthly analgesic consumption (51.1-75.7\%) was inferior to the percentage of patients who reported a $\geq 50 \%$ reduction in MIDAS score (89.5-100\%). HIT-6 score was also consistently reduced at all follow-ups. In patients with a 12-month follow-up, MIDAS and HIT-6 scores were also reduced at all follow-ups compared with baseline, with $84.4-100 \%$ of patients achieving a $\geq 50 \%$ reduction in MIDAS score, and patients with $\mathrm{a} \geq 50 \%$ response rate ranging from 36.4 to $66.6 \%$. No severe adverse events were recorded. Fewer migraine days at baseline were associated with $\geq 50 \%$ response rate at 1 month and fewer MMDs, years of chronic migraine, and monthly analgesic use at 6 months.

Conclusion In resistant chronic migraine patients, anti-CGRP mAbs are effective and safe. A $\geq 50 \%$ reduction in MIDAS score seems to be the most advantageous outcome measure in this setting, which allows most severe migraine patients to persist with treatment.
\end{abstract}

\section{Background}

Pierangelo Geppetti

pierangelo.geppetti@unifi.it

1 Section of Clinical Pharmacology and Oncology, Department of Health Sciences, University of Florence, Florence, Italy

2 Headache Center and Clinical Pharmacology Unit, Careggi University Hospital, Florence, Italy
Migraine is the third most prevalent and the second most disabling disease worldwide in the age range of $20-50$ years, with chronic migraine (CM, $\geq 15$ days per month for at least 3 months) affecting 1.4-2.2\% of the general population [1]. A significant proportion of CM patients have an unsatisfactory response to or do not tolerate pharmacological treatment and, thus, according to the criteria of the European Headache Federation (EHF), fall into the definition of 


\section{Key Points}

Our study provides long-term data on the beneficial class effect of anti-calcitonin gene-related peptide (antiCGRP) monoclonal antibodies in severe drug-resistant patients with chronic migraine and medication overuse.

Our predictors suggest a more likely $\geq 50 \%$ response in patients with lower clinical burden before treatment.

The Migraine Disability Assessment (MIDAS) score is a useful outcome measure, providing a better evaluation of disease burden than monthly migraine days (MMDs) or response rate alone, in this setting.

resistant migraine [2]. These patients have a poor quality of life and a high degree of healthcare resource utilization.

Medication-overuse headache $(\mathrm{MOH})$ is a condition characterized by chronic headache and overuse of different acute medications (for more than 10 or 15 days per month, depending on the medication type) that can paradoxically worsen headache, disability, and quality of life [3].

Monoclonal antibodies (mAbs) that block the calcitonin gene-related peptide (CGRP) or its receptor (anti-CGRP $\mathrm{mAbs}$ ) are a new class of anti-migraine drugs. Three of them, erenumab, galcanezumab, and fremanezumab, have been authorized by the European Medicines Agency (EMA) for preventative treatment of episodic migraine (EM) and CM [4]. Clinical benefits of anti-CGRP mAbs have been proven in 3- to 6-month placebo-controlled randomized clinical trials (RCTs) and in prolonged (9 months to 5 years) open-label extension studies [5, 6]. The majority of real-life studies with anti-CGRP mAbs have collected data up to a 6-month period in a mixed population of EM and CM [5].

Policies for access to novel expensive migraine treatments often entail restrictions that may affect disease management [7-9]. In Italy, the Agenzia Italiana del Farmaco (AIFA; Italian Medicines Agency) policy for covering the cost of anti-CGRP mAbs includes patients with high frequency EM ( $\geq 8$ migraine days per month for at least 3 consecutive months) and previous failure or no tolerability of at least three preventative classes of antimigraine drugs, including tricyclic antidepressants, $\beta$-blockers, neuromodulators, and onabotulinumtoxinA [10]. The criteria for eligibility to the Italian program correspond to those for resistant migraine $[2$, 4]. To maintain patients in the 1 -year reimbursed prescription program, the AIFA requires a $\geq 50 \%$ reduction of the Migraine Disability Assessment (MIDAS) score, assessed at 3 and 6 months of treatment. Furthermore, additional restrictions can be applied on a local basis. A panel of experts of the Tuscany Region health system has recommended that patients with the most severe conditions, such as $\mathrm{CM}$, with or without medication overuse, were treated initially.

Herein, we report a prospective, monocentric, cohort study evaluating the long-term effectiveness and safety of the three anti-CGRP mAbs authorized in Europe in a population of patients that, according to the AIFA rules and regional recommendations, reported $\mathrm{CM}$, failed at least three preventative treatments and, to be granted persistence of treatment, showed a $\geq 50 \%$ reduction of the MIDAS score.

\section{Methods}

All consecutive out-patients treated with anti-CGRP mAbs at the Headache Center, who signed informed consent, were enrolled in the study. Study participants were older than 18 years, with CM according to ICHD-3, with or without medication overuse, and started a preventative therapy with erenumab (70 mg monthly, up to $140 \mathrm{mg}$ ), galcanezumab (240 mg first dose and $120 \mathrm{mg}$ monthly), or fremanezumab (225 mg monthly) from December 2019 to April 2021 at the Headache Center of the Careggi University Hospital, Florence, Italy. The erenumab dose was increased to 140 $\mathrm{mg}$ if an improvement of $<30 \%$ in migraine days reduction occurred.

Treated patients fulfilled the requirements set by the AIFA and by the panel of experts of the Tuscany Region. Patients were informed of the existence of cutoffs that allowed them to continue or not in treatment. However, no detailed information was given on which cutoffs were required to persist in treatment.

All patients had experienced treatment failure for lack of either efficacy or tolerability with three or more different classes of migraine-preventative medications. Efficacy failure was defined as no meaningful improvement in the frequency of headaches, as recommended by the EHF guidelines [4].

During the entire treatment period, patients completed a paper headache diary, recording MMDs, acute medication use, and several questionnaires. A migraine day was defined as a calendar day with a headache meeting criteria for migraine (with or without aura) or a day when an acute migraine-specific medication (triptan or ergot) was used.

Demographics, medical history, migraine characteristics (pain intensity, presence of aura, disease duration, and chronicization onset), previous failure of three or more drug classes including B-blockers, tricyclic antidepressants, antiepileptic drugs, and onabotulinumtoxinA, and failure with other preventative treatments, current concomitant preventative, and acute symptomatic treatments (class and number) were collected at baseline.

Migraine-related clinical burden was assessed with MIDAS and Headache Impact Test 6 (HIT-6) 
questionnaires. The Patient Global Impression of Change (PGIC) was used to assess subjective effectiveness and all adverse events were recorded.

Responders have been defined as those patients with a reduction of $\geq 50 \%$ in MMDs compared to baseline. These patients were further classified into $\geq 50 \%, \geq 75 \%$, and $100 \%$ responders. Patients were instructed to report any adverse drug reaction (ADR) throughout the study, reporting mild events in a paper diary, and to promptly communicate moderate and severe ADRs to investigators.

The study was performed according to the Strengthening the Reporting of Observational Studies in Epidemiology (STROBE) guidelines. The study was approved as a part of the Registro Italiano Cefalee (RICe) study by the local ethics committee (Studio RICe, 14591_oss).

\subsection{Outcomes}

All patients that started treatment, regardless of follow-up data, have been included in the safety analysis. Primary outcomes were reduction from baseline in MIDAS score and MMDs, as well as response rates $(\geq 50 \%, \geq 75 \%$, and $100 \%$ reduction in MMDs) during treatment with antiCGRP mAbs. Additional effectiveness outcomes were reduction from baseline of the overall number of acute medications per month and days with at least one acute medication use. Changes from baseline in the HIT-6 questionnaire total scores and overall improvement assessed with the PGIC scale were also measured. Patients with medication overuse did not undergo detoxification intervention prior to or during anti-CGRP mAbs. The percentage of patients who maintained the medication overuse was calculated at each follow-up. A sub-analysis to evaluate responder rate and MMDs was conducted in patients previously treated with onabotulinumtoxinA to evaluate indirect effectiveness in patients that failed this treatment. An additional effectiveness analysis has been performed in the subgroup of patients who completed the 12-month follow-up (with and without dropouts).

\subsection{Statistical Analysis}

The sample size was not based on any statistical considerations. Effectiveness analysis included two population sets: patients with at least 1 month of follow-up (including all patients with $\geq 1$ month of follow-up, $n=184$ ) and patients who completed 12 months of treatment with any of the mAbs $(n=52)$ (see Fig. 1). No patients refused to participate or withdrew informed consent during the study. Demographic and baseline characteristics were summarized descriptively. A simple explorative descriptive analysis was performed to evaluate outcomes with each $\mathrm{mAb}$, considering that the study was not designed to assess differences among mAbs.

Normality assumption was assessed using the ShapiroWilk test. Wilcoxon signed-rank test was calculated to study effectiveness variables pre-post changes in quantitative variables. For independent variables, the Mann-Whitney $U$ test for continuous variables and the two-tailed Pearson chi-squared test or the Fisher's test for categorical variables were applied as appropriate. Finally, univariate and multivariate logistic regression analysis was carried out (odds ratios [ORs] and 95\% confidence intervals [CIs]) to explore the variables independently associated with responder status at 1 and 12 months; variables included in the equation were significant in previous analysis or had a clinical interest. The good fit of the logistic regression model was determined by using the Hosmer-Lemeshow test. A $p$ value $<0.05$ was considered significant for all variables. All the data were analyzed using SPSS software version 26.0 (IBM Corp. SPSS Statistics, Armonk, NY, USA).

\section{Results}

As of April 2021, 203 patients (78.3\% females, mean age $49.4 \pm 12.2$ years) with $\mathrm{CM}$ had started an anti-CGRP mAb as a preventative treatment for migraine and were included in the safety analysis. Overall, $47.3 \%$ of patients (96/203) were treated with erenumab, which entered the national/ regional prescription program first, $36.5 \%$ (74/203) with galcanezumab, which entered at a later stage, and $16.3 \%$ (33/203) with fremanezumab, which was the last to be introduced. At the time of the analysis, 90.6\% (184/203) of patients had completed at least 1 month of treatment and were included in the effectiveness analysis with a mean (SD) treatment duration of 6.4 (3.9) months. Moreover, 52 patients (25.6\%) completed a 12-month follow-up, whereas 33 of those patients completed a 12-month treatment (Fig. 1 and Table 1). Of the initial cohort of patients, $59.6 \%(31 / 52)$ received erenumab (70 or $140 \mathrm{mg}$ ), 34.6\% (18/52) galcanezumab, and 5.8\% (3/52) fremanezumab. Demographic and baseline headache characteristics are reported in Table 1

Most patients $(84.8 \%, 172 / 203)$ reported medication overuse. A failure of four or more prior preventative treatment classes was reported by a high number $(82.3 \%$, $167 / 203)$ of patients, and $22.2 \%(45 / 203)$ were on a preventative concomitant medication (excluding onabotulinumtoxinA, not allowed as co-treatment by the AIFA prescription program) when they started the anti-CGRP mAbs treatment (Table 1). At baseline, the mean headache pain intensity on a $0-10$ scale (numeric rating scale [NRS]) was $7.9 \pm 1.3$ points, days per month with at least one analgesic use was $21.3 \pm 8.2$, and the median (interquartile range [IQR]) number of acute medication use was 30 [33] per 


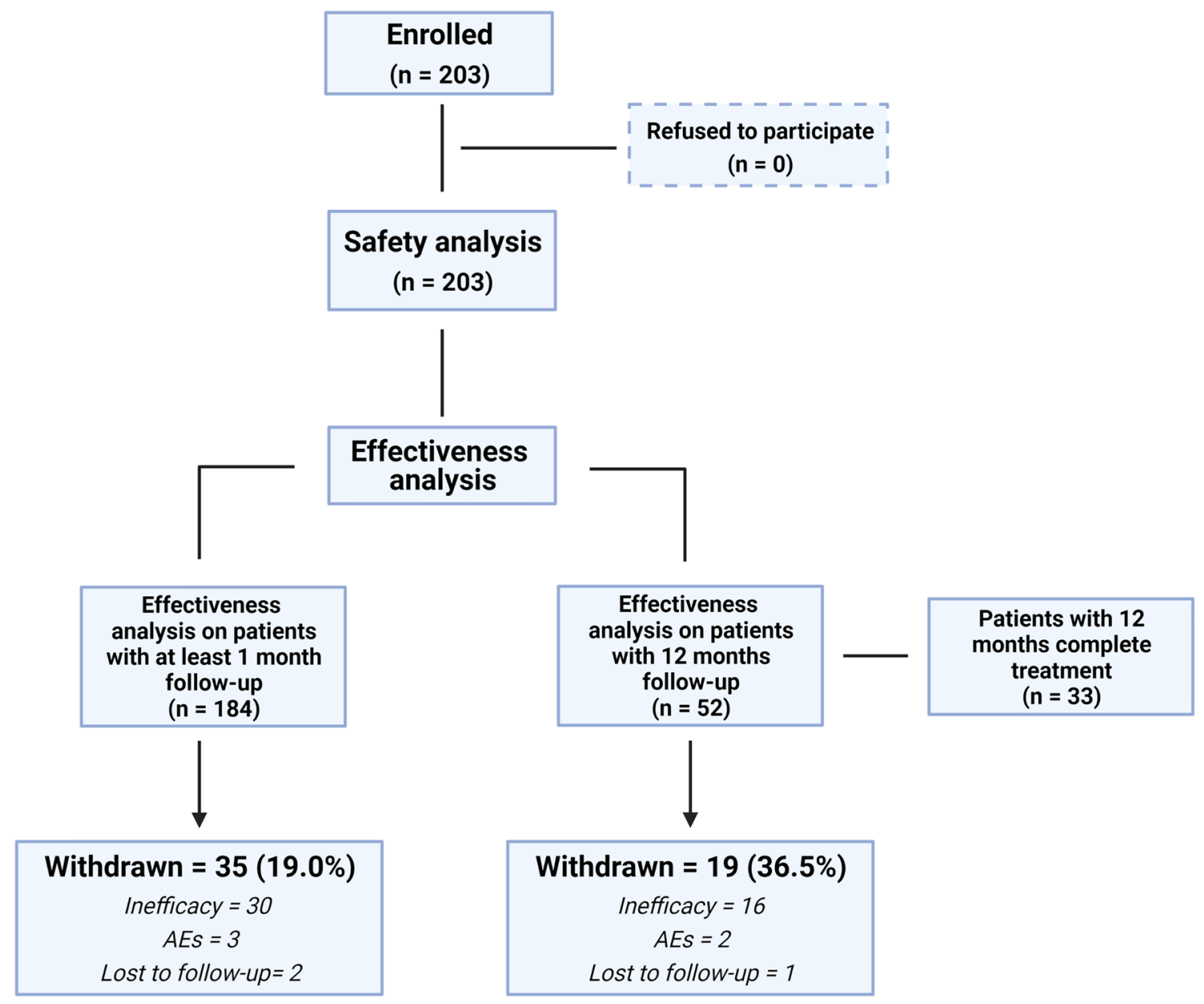

Figure 1. Patient distribution flow-chart in effectiveness and safety analysis. $A E$ adverse event

month. Patients presented a high MIDAS $(101.3 \pm 63.7)$ and HIT-6 score $(67.9 \pm 5.6)$ at baseline. Overall, $19.0 \%$ of migraineurs (35/184) dropped out of treatment for lack of efficacy (30/35 [85.7\%]) as self-reported by patient and/ or according to physician decision, adverse events $(3 / 35$ [8.6\%]), or lost to follow-up (2/35 [5.7\%]).

\subsection{Treatment Effectiveness Analysis}

Overall, the MIDAS score was markedly attenuated ( $p<$ 0.0001 ) (Fig. 2a), and a high percentage of patients showed a $\geq 50 \%$ reduction in MIDAS score, which varied between 89.5\% (at 6 months) and 100\% (at 12 months) (Fig. 2b). Treatment was withdrawn in 16 out of 35 patients (five at month 3 , ten at month 6 , one at month 9 , and none at month 12 ) due to a $<50 \%$ reduction in the MIDAS score (Fig. 2b). MMD decrease ranged from 8.2 to 12.1 from baseline $(p<0.0001)$ (Fig. 3a). Further reductions in MMDs were observed at 3 and 6 months ( $p<0.0001$ ), but not at 12 months, compared with 1 month of follow-up. Reduction in acute medication consumption was reported at all months, both as the total number of analgesics used per month, ranging to 16.7 from $27.7(p<0.0001)$ (reduction $\geq 50 \%$ from $51.1 \%$ to $75.7 \%$ ), and days with at least one analgesic use, ranging to 8.1 from $12.4(p<0.0001)$ (Fig. $3 \mathrm{~b}$ and Table $\mathrm{S} 1$ in the electronic supplementary material). Reduction in the number of acute medications per month was similar for all four pharmacological classes of analgesics (Figure S1, see the electronic supplementary material). Patients with medication overuse dropped from $84.8 \%$ before treatment to a range of $24.2 \%$ to $37.5 \%$ after treatment (Table S2, see the electronic supplementary material). Patients with a $\geq 50 \%$ response rate (reduction in MMDs) ranged from 36.4 to $56.8 \%$, whereas $\geq 75 \%$ of the responders ranged from $13.6 \%$ to $33.0 \%$ (Fig. 4a). At months 1, 6, and 9, between four out of $94(4.3 \%)$ and two out of $62(3.2 \%)$ patients became attack 
Table 1. Patients demographic and clinical features

\begin{tabular}{lll}
$\begin{array}{l}\text { Safety analysis } \\
(n=203)\end{array}$ & Effectiveness analysis & Effectiveness analysis \\
& At least 1 month & 12 months \\
$(n=184)$ & $(n=52)$ \\
\hline
\end{tabular}

\section{Demographics}

Age [years], mean $\pm \mathrm{SD}$

Sex female, $n(\%)$

\begin{tabular}{|c|c|c|}
\hline $49.4 \pm 12.2$ & $48.2 \pm 12.6$ & $45.5 \pm 14.7$ \\
\hline $159(78.3)$ & $145(78.8)$ & $46(88.5)$ \\
\hline $24.1 \pm 5.9$ & $24.8 \pm 5.7$ & $25.4 \pm 5.5$ \\
\hline $14(6.9)$ & $13(7.1)$ & $3(5.8)$ \\
\hline $32.9 \pm 13.2$ & $32.4 \pm 12.8$ & $32.1 \pm 13.9$ \\
\hline $16.1 \pm 11.5$ & $15.6 \pm 11.4$ & $18.2 \pm 11.3$ \\
\hline $7.9 \pm 1.3$ & $7.9 \pm 1.2$ & $7.8 \pm 0.9$ \\
\hline $0.3(0.6)$ & $0.3(0.6)$ & $0.3(0.7)$ \\
\hline $4.4 \pm 0.8$ & $4.4 \pm 0.8$ & $4.6 \pm 0.6$ \\
\hline $172(84.7)$ & $156(84.8)$ & $52(100.0)$ \\
\hline $21.2 \pm 8.1$ & $21.3 \pm 8.2$ & $24.0 \pm 6.2$ \\
\hline $30(33)$ & $30(34)$ & $37(32)$ \\
\hline $101.3 \pm 63.7$ & $102.9 \pm 64.9$ & $139.2 \pm 68.7$ \\
\hline $67.9 \pm 5.6$ & $67.7 \pm 5.3$ & $69.9 \pm 3.4$ \\
\hline $60(29.6)$ & $54(16.8)$ & $10(19.2)$ \\
\hline $107(52.7)$ & $99(53.8)$ & $37(71.1)$ \\
\hline 190 (93.6) & 171 (92.9) & $48(92.3)$ \\
\hline 192 (94.6) & $173(94.0)$ & $48(92.3)$ \\
\hline $171(84.2)$ & $158(85.9)$ & $47(90.4)$ \\
\hline $187(92.1)$ & $169(91.8)$ & $49(94.2)$ \\
\hline $28(13.8)$ & $25(13.6)$ & $7(13.5)$ \\
\hline $126(62.1)$ & 117 (63.6) & $42(80.8)$ \\
\hline
\end{tabular}

\section{Migraine features}

Monthly migraine days, mean $\pm \mathrm{SD}$

Aura, $n(\%)$

Migraine duration (years), mean $\pm \mathrm{SD}$

Chronicization duration (years), mean \pm SD

NRS score, mean $\pm \mathrm{SD}$

Concomitant preventive treatment, mean (SD)

Prior preventive classes failures, mean $\pm \mathrm{SD}$

Medication overuse, $n(\%)$

Days in previous month with at least one analgesic use, mean \pm SD

Analgesic doses taken in previous month, median (IQR)

\section{Migraine-related clinical burden}

Disability (MIDAS), mean \pm SD

Headache-related impact (HIT-6), mean \pm SD

Prior preventive class failures*

4 classes

$>4$ classes

Drug classes

Beta-blockers

Tricyclic antidepressant

Calcium channel blockers

Antiepileptic drugs

SSRI/SNRI

OnabotulinumtoxinA

Percentages are expressed on column total

HIT-6 Headache Impact Test 6, IQR interquartile range, MIDAS Migraine Disability Assessment, NRS numeric rating scale, $S D$ standard deviation, SNRI serotonin-norepinephrine reuptake inhibitor, SSRI selective serotonin reuptake inhibitor

* All patients have at least 3 prior preventive class failures

free (100\% responders, Fig. 4a). HIT-6 and self-measured improvement with the PGIC scale showed a significant reduction compared to baseline $(p<0.0001)$ (Figure $\mathrm{S} 2$, see the electronic supplementary material).

In patients with a 12-month follow-up (52/184), clinical outcomes were assessed first in the cohort that continued with treatment for 12 months (33/52 [63.5\%]; 19 dropouts) due to clinical benefit. Reduction in MMDs from baseline ranged from 8.4 to $13.2(p<0.0001)$ (Figure S3a, see the electronic supplementary material); MIDAS and HIT-6 scores were also reduced at all follow-ups compared with baseline (Figure S4). Analgesic consumption decreased from baseline both in absolute number of analgesics used and days with at least one analgesic use (Figure $\mathrm{S} 3 \mathrm{~b}$ ). Patients with $\mathrm{a} \geq 50 \%$ response rate ranged from
36.4 to $66.6 \%$ (Fig. 4 b), whereas $84.4-100 \%$ of patients achieved a $\geq 50 \%$ reduction in MIDAS score. In addition, to avoid effectiveness overestimation, the $\geq 50 \%$ reduction in MIDAS score and response rate were calculated in all patients, including those who dropped out during the 1 year of treatment (52/184). To perform this calculation, $\mathrm{a}<50 \%$ reduction in MIDAS score and response rate was always applied to dropout patients. Patients with a $\geq$ $50 \%$ response rate ranged from 32.7 to $42.3 \%$ (Figure $4 \mathrm{c}$ ), whereas those with a $\geq 75 \%$ response rate ranged from 15.4 to $26.9 \%$. No patients achieved a $100 \%$ response in this cohort. Patients that achieved a reduction $\geq 50 \%$ in MIDAS score ranged from 63.5 to $96.1 \%$.

Differences at baseline in patient subpopulations treated with or naïve to onabotulinumtoxinA are reported in 
Figure 2. Migraine Disability Assessment (MIDAS) questionnaire total scores (A) and number [percentage] of patients who persisted with treatment based on $\geq 50 \%$ reduction in MIDAS score (B) during treatment follow-up. Error bars represent 95\% confidence intervals. Number in square represents mean reduction compared to baseline within the group for each follow-up (A) or percentage of patients (B) per group

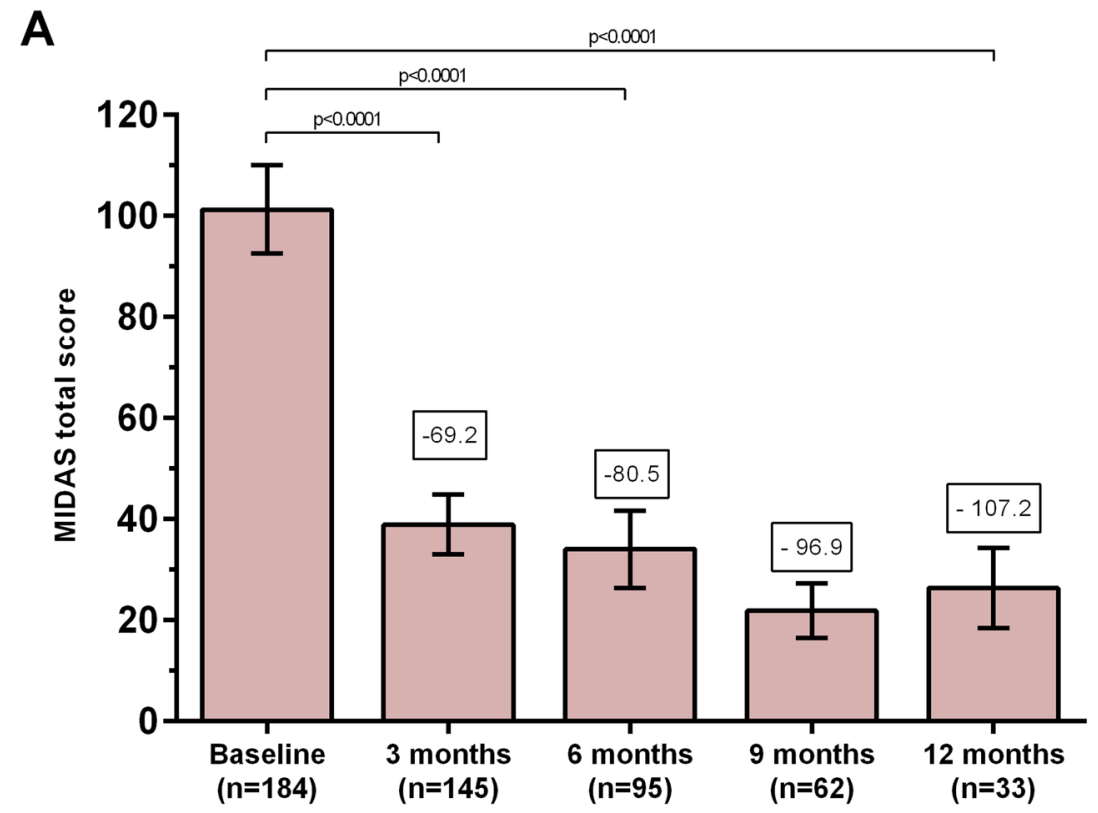

B

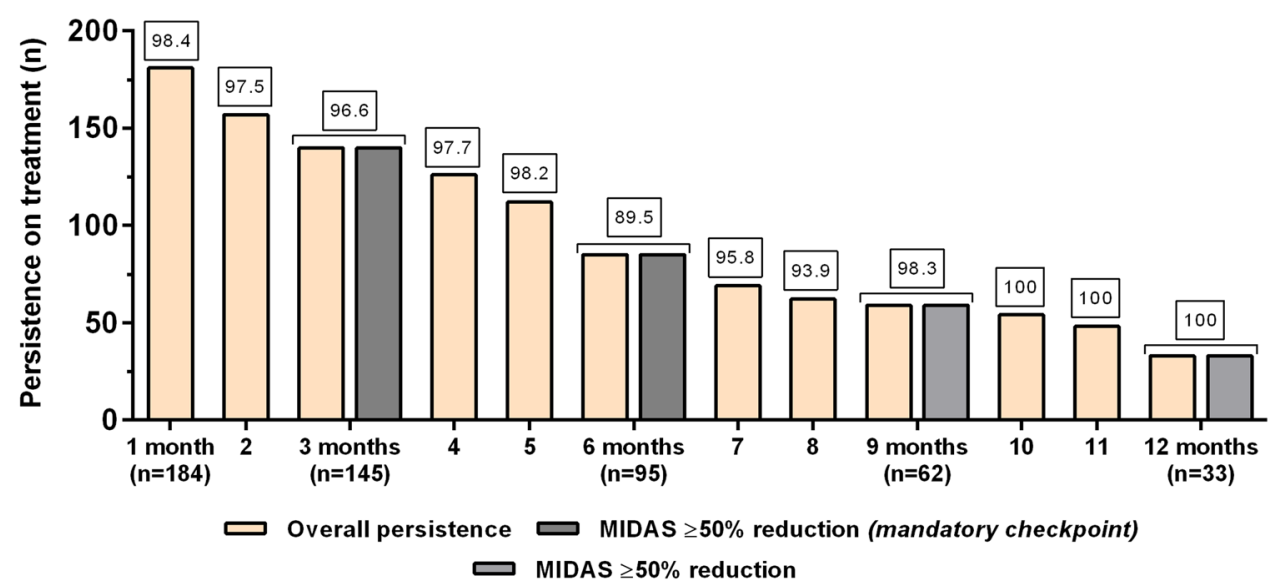

Table S2 (see the electronic supplementary material). No differences in response rates have been observed between naïve patients and patients who failed onabotulinumtoxinA treatment (Tables 2 and 3). Finally, the explorative descriptive analysis of the three anti-CGRP mAbs of MIDAS score, MMDs, and responder rates is reported in Figure S5 (see the electronic supplementary material).

\subsection{Clinical Predictors of Response}

Fewer migraine days at baseline were associated with $\geq 50 \%$ response rate at 1 month and fewer MMDs, years of CM, and monthly analgesic use at 6 months (Table 2). Univariate and multivariate analyses were performed to assess clinical predictors of $\geq 50 \%$ response in patients with 1,3 , and 6 months of follow-up (Table 3). In the univariate analysis, duration of chronicization (OR 0.96; 95\% CI 0.93-0.99, $p=0.04$ ) and an elevated number of MMDs at baseline (OR 0.90; 95\% CI $0.85-0.95, p<0.0001$ ) were independently associated with a lower response rate at 1 month and 6 months. MMDs at baseline (OR 0.87; 95\% CI 0.80-0.97, $p=0.002$ ), duration of chronicization (OR 0.95 ; 95\% CI 0.92-0.99, $p=0.04$ ), and total number of analgesics (OR 0.97 ; 95\% CI 0.96-0.99, $p=0.003$ ) were associated with a lower responder rate at 6 months, confirming predictors identified at 1 month.

Multivariate logistic regressions using the variables included in the univariate analysis were performed. The models explained $20 \%, 11 \%$, and $30 \%$ (Nagelkerke $R^{2}$ ) of the variance to achieve responder status at 1,3 , and 6 months. Age was associated with higher response rate (OR 1.06; 95\% CI 1.01-1.10; $p=0.02$ ), whereas MMDs at baseline were independently associated (as in univariate regression) with a 

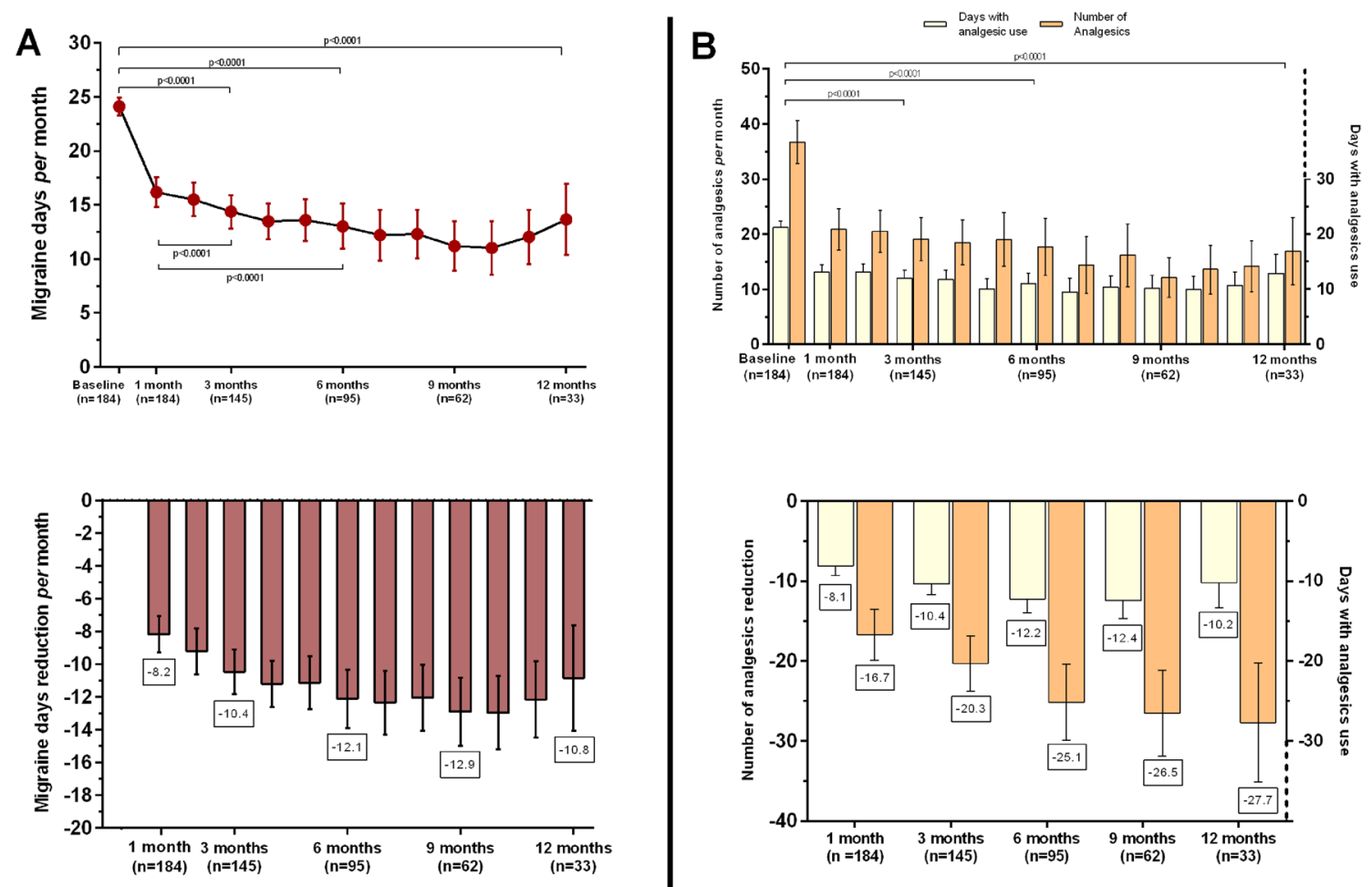

Figure 3. A Monthly migraine days (MMDs) frequency during antiCGRP treatment in patients with at least 1-month follow-up and migraine days at each follow-up and MMD reduction compared to baseline. B Number of analgesics used and days with at least one analgesic used per month and their reduction compared to baseline.
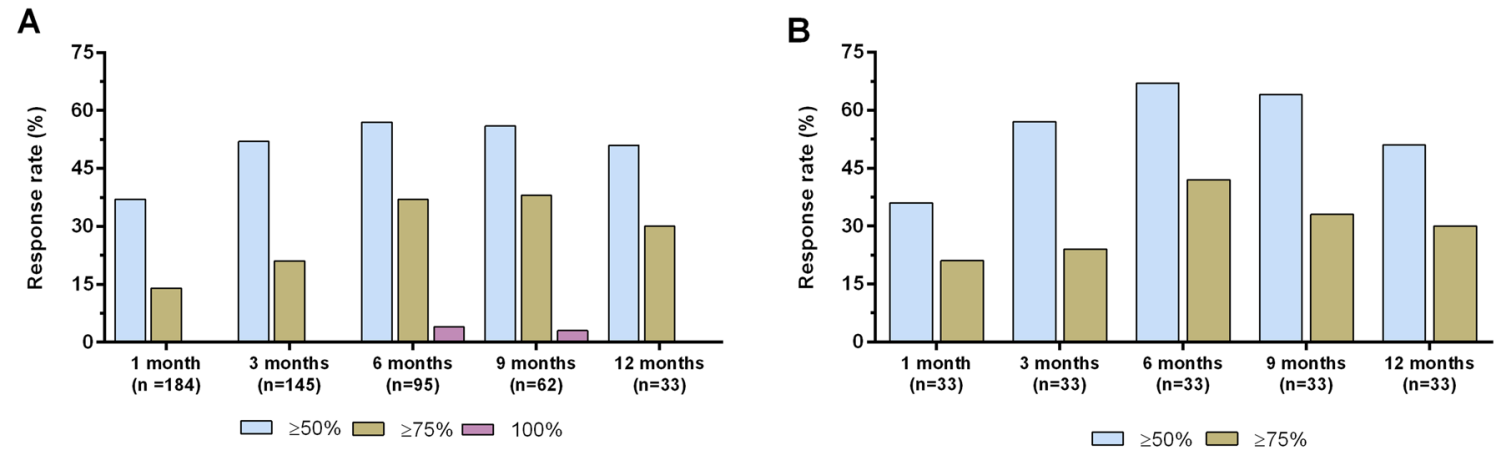

Error bars represent 95\% confidence intervals. Analgesics include NSAIDs, triptans, analgesic associations (with or without opioids), opioids, and ergot derivative. CGRP calcitonin gene-related peptide, NSAID nonsteroidal anti-inflammatory drug

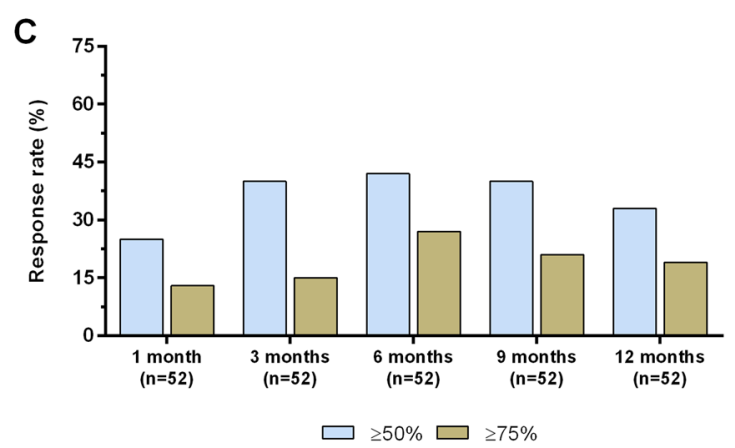

Figure 4. A Effectiveness analysis of response rate $\geq 50 \%, \geq 75 \%$, and $100 \%$ in all patients; B in patients that completed 12 months of treatment; and $\mathbf{C}$ in patients with 12-month follow-up 
Table 2. Analysis of baseline variables associated to responders $\geq 50 \%$ after 1,3 , and 6 months of treatment

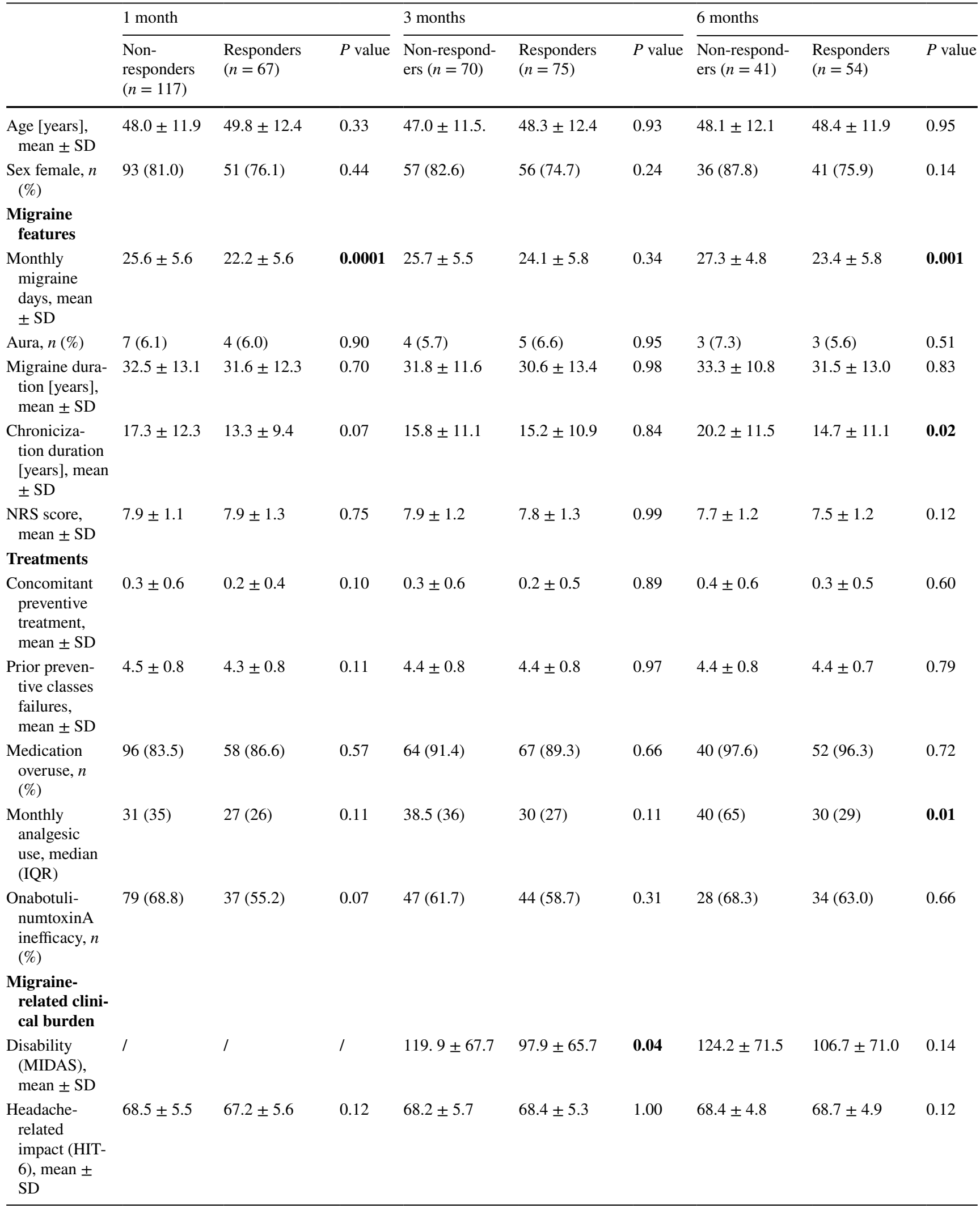

Values in bold are statistically significant; percentages are expressed on column total

$I Q R$ interquartile range, HIT-6 Headache Impact Test 6, MIDAS Migraine Disability Assessment, NRS numeric rating scale, SD standard deviation 
Table 3. Univariable and multivariable regressions with selected variables for clinical response $\geq 50 \%$

\begin{tabular}{|c|c|c|c|c|c|c|c|c|c|}
\hline & \multicolumn{3}{|c|}{1 month } & \multicolumn{3}{|c|}{3 months } & \multicolumn{3}{|c|}{6 months } \\
\hline & OR & $95 \% \mathrm{CI}$ & $P$ value & OR & $95 \% \mathrm{CI}$ & $P$ value & OR & $95 \% \mathrm{CI}$ & $P$ value \\
\hline \multicolumn{10}{|l|}{ Univariable } \\
\hline Age (years) & 1.01 & $0.98-1.04$ & 0.33 & 1.01 & $0.98-1.04$ & 0.53 & 1.01 & $0.96-1.04$ & 0.91 \\
\hline Sex, female & 0.76 & $0.36-1.58$ & 0.46 & 0.61 & $0.27-1.37$ & 0.23 & 0.44 & $0.14-1.34$ & 0.15 \\
\hline Migraine duration (years) & 0.99 & $0.97-1.02$ & 0.67 & 0.99 & $0.96-1.02$ & 0.59 & 0.98 & $0.95-1.02$ & 0.51 \\
\hline Chronicization duration (years) & 0.96 & $0.93-0.99$ & 0.04 & 0.99 & $0.96-1.03$ & 0.74 & 0.95 & $0.92-0.99$ & 0.04 \\
\hline Medication overuse & 0.78 & $0.33-1.85$ & 0.58 & 0.78 & $0.25-2.39$ & 0.67 & 0.65 & $0.05-7.42$ & 0.72 \\
\hline Baseline migraine days & 0.90 & $0.85-0.95$ & $<0.0001$ & 0.95 & $0.89-1.01$ & 0.10 & 0.87 & $0.80-0.95$ & 0.002 \\
\hline Total number of analgesics & 0.98 & $0.97-1.00$ & 0.06 & 0.98 & $0.97-1.00$ & 0.05 & 0.97 & $0.96-0.99$ & 0.003 \\
\hline MIDAS score & l & / & l & 0.99 & $0.99-1.00$ & 0.05 & 0.99 & $0.99-1.00$ & 0.21 \\
\hline HIT-6 score & 0.95 & $0.90-1.01$ & 0.12 & 1.01 & $0.94-1.06$ & 0.82 & 1.01 & $0.93-1.10$ & 0.75 \\
\hline Prior onabotulinumtoxinA treatment & 1.77 & $0.95-3.31$ & 0.07 & 0.69 & $0.35-1.37$ & 0.29 & 0.79 & $0.33-1.86$ & 0.58 \\
\hline \multicolumn{10}{|l|}{ Multivariable } \\
\hline Age (years) & 1.06 & $1.01-1.10$ & 0.02 & 1.03 & $0.97-1.08$ & 0.26 & 1.05 & $0.96-1.14$ & 0.22 \\
\hline Sex, female & 0.66 & $0.27-1.66$ & 0.38 & 0.83 & $0.29-2.32$ & 0.72 & 0.39 & $0.06-2.40$ & 0.31 \\
\hline Migraine duration (years) & 0.96 & $0.91-1.02$ & 0.24 & 0.95 & $0.89-1.02$ & 0.18 & 0.96 & $0.86-1.06$ & 0.43 \\
\hline Chronicization duration (years) & 0.96 & $0.92-1.01$ & 0.13 & 1.02 & $0.97-1.07$ & 0.42 & 0.97 & $0.90-1.03$ & 0.33 \\
\hline $\mathrm{MOH}$ & 1.24 & $0.45-3.45$ & 0.66 & 1.03 & $0.27-3.85$ & 0.96 & 1.07 & $0.07-16.1$ & 0.96 \\
\hline Baseline migraine days & 0.92 & $0.86-0.98$ & 0.02 & 0.96 & $0.88-1.04$ & 0.29 & 0.91 & $0.81-1.01$ & 0.07 \\
\hline Total number of analgesics & 0.99 & $0.98-1.01$ & 0.85 & 0.99 & $0.98-1.00$ & 0.59 & 0.98 & $0.96-1.01$ & 0.21 \\
\hline MIDAS score & l & l & l & 0.99 & $0.98-1.00$ & 0.05 & 0.99 & $0.98-1.01$ & 0.27 \\
\hline HIT-6 score & 0.95 & $0.87-1.03$ & 0.25 & 1.08 & $0.98-1.18$ & 0.10 & 1.09 & $0.93-1.28$ & 0.25 \\
\hline Prior onabotulinumtoxinA treatment & 1.02 & $0.47-2.19$ & 0.95 & 1.02 & $0.43-2.36$ & 0.95 & 2.29 & $0.65-8.01$ & 0.19 \\
\hline
\end{tabular}

Values in bold are statistically significant

CI confidence interval, HIT-6 Headache Impact Test 6, MIDAS Migraine Disability Assessment, $M O H$ Medication-overuse headache, $O R$ Odds Ratio

lower responder rate (OR 0.92; 95\% CI 0.86-0.98, $p=0.02$ ) at 1 month (Table 3 ). No variables have been significantly associated at 3 and 6 months.

\subsection{Safety and Tolerability Analysis}

No severe treatment-related adverse events have been reported throughout the study. A total of 16 patients $(7.9 \%)$ experienced at least one ADR, with 23 ADRs overall reported (Table S3, see the electronic supplementary material). The most common were fatigue (3.9\%), injection site erythema $(1.5 \%)$, constipation $(1.5 \%)$, and muscle pain (1.5\%). Only three patients $(1.5 \%)$ withdrew from antiCGRP mAb treatment due to adverse events, in particular fatigue - two after 1 month and one after 4 months of treatment.

\section{Discussion}

Few observational studies on long-term effectiveness of antiCGRP mAbs and no merged results of three mAbs have been reported [8, 11-16]. Most observational studies, with follow-up ranging from 3 to 6 months, enrolled patients with erenumab [8, 11-15, 17-21]; one study included patients treated with erenumab and galcanezumab [9], and another one with only galcanezumab [22]. Three prolonged (12-month follow-up) real-world studies were limited to erenumab [15, 23, 24]. A 52-week open-label study with erenumab reported sustained effectiveness (-9.3 MMDs) at 12 months in a subgroup of patients with CM [25]. A communication on galcanezumab in a 12-month open-label study reported a 8.0-9.0 reduction in monthly headache days (MHDs) and a responder rate of 53.3-56.9\% at 12 months [26]. Of note, no long-term open-label extension studies have been published so far with fremanezumab.

We report the long-term effectiveness and tolerability of all three anti-CGRP antibodies authorized by EMA for migraine, prescribed according to both the AIFA criteria and the expert panel recommendations of the Tuscany Region. 
According to these criteria, only resistant CM patients [2], most with medication overuse, were treated, and continuation of the prescription was allowed only if patients reported a reduction of $\geq 50 \%$ in MIDAS score at 3 and 6 months. Our results indicate that anti-CGRP $m A b s$ are effective and well tolerated over a period of 12 months of treatment in resistant patients with $\mathrm{CM}$ and medication overuse. In these severe patients, a significant reduction in MMDs and other efficacy outcomes was observed at the first month of treatment, followed by a further slight decrease until month 6 (Table 3; Figures 2, 3, and 4), without any additional benefits observed in the overall population (Figure 2 ) and in the sub-analysis of patients who completed the 12-month treatment (Figure S3a, see the electronic supplementary material). This could suggest that CGRP has only a partial role in migraine pathogenesis and/or only a fraction of CGRP signaling can be counteracted by the mAbs in a majority of migraineurs. Further, the requirement of 6 months to reach the therapeutic plateau could suggest that a prolonged period is necessary to counteract the neurobiological mechanisms underpinning CGRP desensitization. The MIDAS score dropped at month 3 and the reduction persisted until month 12. The MIDAS questionnaire was developed to measure headache-associated disability and improve communication between physicians and patients [27]. Although its use does not exclude the usefulness of diaries to avoid underestimation of headache intensity and overestimation of headache frequency [28], the MIDAS questionnaire has demonstrated reliability [27], and MIDAS score is highly correlated with physician judgment about the severity of disease [28]. Here, we report that the use of the MIDAS score provided a better evaluation of the disease burden than MMDs or response rate alone in this setting, detecting amelioration and allowing the continuation of treatment for a large proportion of patients.

In our overall effectiveness population, responder rates are in line with two recent observational studies [9, 29], but are lower compared to other studies that enrolled CM patients treated with erenumab, which ranged from 53 to $69.7 \%$ at 3 months, $46.5 \%$ to $70 \%$ at 6 months $[11,14,21$, 22], and $75.6 \%$ at 1 year [24]. To limit the overestimation inherent to the ongoing design of the present study, we performed the responder rate analysis in patients with 12 -month follow-up. In this cohort $(n=52)$, the $\geq 50 \%$ response rate was slightly lower (about 40\%) than that found in the general cohort. However, this difference could depend on the higher clinical burden of patients who first entered our prescription program. Notably, a study evaluating erenumab in $\mathrm{CM}$ patients that failed five preventive medications [13] reported a responder rate at 3 months considerably lower (31.3\%) than other studies [11, 14, 21 , 22], and similar to our 12-month follow-up cohort and the post hoc analysis of CM resistant patients in the erenumab phase II trial (31.1-38.5\%) [30], highlighting differences between populations with different profiles of resistance to treatments.

A proportion of patients who failed treatment with onabotulinumtoxinA has been analyzed separately (Table 2 , S2, see the electronic supplementary material). No difference was found in the percentage of responders between patients naïve to onabotulinumtoxinA and patients who failed onabotulinumtoxinA treatment (Table 2), in line with prior studies [13, 14]. Although several confounding factors may exist, these results suggest that the antimigraine action of onabotulinumtoxinA may not reside in an efficient inhibition of CGRP release [31], and to further explore the combination of onabotulinumtoxinA and anti-CGRP mAbs in refractory patients [32].

Patients with medication overuse, although receiving advice to limit the consumption of acute medications, did not undergo any specific detoxification intervention prior to or during anti-CGRP mAbs treatment. A similar reduction in outcome measures was observed in medication-overuse and non-medication-overuse patients (Table 2). These data are in line with recent open-label studies $[9,14,29]$ and a subgroup analysis of one RCT, and with results from administrative databases [33,34]. In addition to the commonly used measure for medication overuse, we evaluated the absolute number of analgesics per month. Anti-CGRP mAbs reduced the overall number of acute medications as well as the different classes of anti-migraine medicines.

There is no current evidence for robust clinical predictors of response to anti-CGRP mAbs. An analysis comparing baseline features of $\geq 50 \%$ responders and not responders at different follow-ups (short-term and long-term response) and multi-variable regression analysis showed that patients with lower MMDs at baseline were more likely to achieve a $\geq 50 \%$ response at 1 and 6 months. Contrary to some studies that have reported a positive association between MMDs at baseline and response rate $[9,15,24]$, but in line with other studies [11, 14, 35], our predictors suggest a more likely $\geq$ $50 \%$ response in patients with lower clinical burden before treatment.

No serious adverse reactions occurred during treatment, and few adverse events were reported, with lower rates compared to other observational studies and clinical trials [36]. Only three patients discontinued treatment due to ADRs (i.e., persistent fatigue), whereas constipation was mild and controlled with dietary changes or supplements.

The 12-month follow-up and evaluation of different subpopulations and parameters, not assessed in previous studies (number of analgesics per month subdivided by pharmacological class) (Figure S4, see the electronic supplementary material) are the strengths of this study. In addition, requirements set by the AIFA and Tuscany Region experts for the access and continuation in the reimbursed prescription 
program of three different anti-CGRP mAbs are unique features of the study that reflect a specific clinical practice. A limitation of this study, in addition to its observational nature, is the lack of a simultaneous cohort for comparison. However, it should be noted that patients with long-term $\mathrm{CM}$ that have failed numerous preventive treatments are less inclined to a placebo effect [13]. Moreover, the use of only MMDs without MHDs could be a limitation. Finally, we did not consistently assess the effect of withdrawal and reintroduction of concurrent preventive treatments; however, this condition applied to only a few patients.

\section{Conclusion}

Observational, real-world studies are required to optimize the use of treatments in multifaceted clinical settings. Our study has demonstrated a beneficial class effect of antiCGRP mAbs in severe drug-resistant patients with $\mathrm{CM}$ and medication overuse. Although further studies are necessary to reliably identify predictors of response, a higher number of MMDs at baseline seems to indicate an inferior response. As the $\geq 50 \%$ reduction in MIDAS score was met by a larger number of patients than other outcome measures, the AIFA mandate to link prescription to the MIDAS score results to be the most advantageous choice in this setting, allowing more patients to continue with treatment.

Supplementary Information The online version contains supplementary material available at https://doi.org/10.1007/s40263-021-00893-y.

\section{Declarations}

Funding Open access funding provided by Università degli Studi di Firenze within the CRUI-CARE Agreement. This research did not receive any specific grants from funding agencies in the public, commercial, or not-for-profit sectors.

Conflict of interest PG received personal fees from Allergan, Eli Lilly, Novartis, Amgen, and TEVA and grants from Amgen, TEVA, Eli-Lilly, Allergan, and Chiesi; was involved in the Scientific Advisory Board, Endosome Therapeutics; and is the founding scientist of FloNext srl, Spinoff of the University of Florence. FDC received personal fees from TEVA, Eli Lilly, and Novartis. LFI, DF, SB, and AC declare no conflicting interests.

Data availability The data collected and analyzed for the current study are available from the corresponding author on reasonable request.

Ethics The study was approved as a part of the Registro Italiano Cefalee (RICe) study by the local Ethics committee (Studio RICe, 14591_oss).

Consent to Participate Informed consent was obtained from every patient.
Consent to Publish Not applicable.

Code availability Not applicable.

Authors' Contributions PG, LFI, and FDC designed the study; LFI, DF, SB, AC, FDC, and PG performed the research; and LFI, PG, and FDC analyzed and interpreted the data. PG, DF, SB, AC, and FDC critically revised the draft manuscript. All authors critically reviewed the manuscript, agree to be fully accountable for ensuring the integrity and accuracy of the work, and read and approved the final manuscript.

Open Access This article is licensed under a Creative Commons Attribution-NonCommercial 4.0 International License, which permits any non-commercial use, sharing, adaptation, distribution and reproduction in any medium or format, as long as you give appropriate credit to the original author(s) and the source, provide a link to the Creative Commons licence, and indicate if changes were made. The images or other third party material in this article are included in the article's Creative Commons licence, unless indicated otherwise in a credit line to the material. If material is not included in the article's Creative Commons licence and your intended use is not permitted by statutory regulation or exceeds the permitted use, you will need to obtain permission directly from the copyright holder. To view a copy of this licence, visit http://creativecommons.org/licenses/by-nc/4.0/.

\section{References}

1. Ashina M. Migraine. N Engl J Med. 2020;2020(383):1866-76.

2. Sacco S, Braschinsky M, Ducros A, Lampl C, Little P, van den Brink AM, et al. European headache federation consensus on the definition of resistant and refractory migraine: developed with the endorsement of the European Migraine \& Headache Alliance (EMHA). J Headache Pain. 2020;2020(21):76.

3. Kristoffersen ES, Lundqvist C. Medication-overuse headache: epidemiology, diagnosis and treatment. Ther Adv Drug Saf. 2014;2014(5):87-99.

4. Sacco S, Bendtsen L, Ashina M, Reuter U, Terwindt G, Mitsikostas DD, Martelletti P. European headache federation guideline on the use of monoclonal antibodies acting on the calcitonin gene related peptide or its receptor for migraine prevention. J Headache Pain. 2019;2019(20):6.

5. Mavridis T, Deligianni CI, Karagiorgis G, Daponte A, Breza M, Mitsikostas DD. Monoclonal antibodies targeting CGRP: from clinical studies to real-world evidence-what do we know so far? Pharmaceuticals. 2021;14:2021.

6. Ashina M, Goadsby PJ, Reuter U, Silberstein S, Dodick DW, Xue $F$, et al. Long-term efficacy and safety of erenumab in migraine prevention: Results from a 5-year, open-label treatment phase of a randomized clinical trial. Eur J Neurol. 2021;2021(28):1716-25.

7. Cape S. Access to migraine treatments in Ontario, Canada: a review of the ontario drug benefit program. Headache. 2020;2020(60):1888-900.

8. Lambru G, Hill B, Murphy M, Tylova I, Andreou AP. A prospective real-world analysis of erenumab in refractory chronic migraine. J Headache Pain. 2020;2020(21):61.

9. Torres-Ferrus M, Gallardo VJ, Alpuente A, Caronna E, GineCipres E, Pozo-Rosich P. The impact of anti-CGRP monoclonal antibodies in resistant migraine patients: a real-world evidence observational study. J Neurol. 2021.

10. Attivazione web e pubblicazione schede di monitoraggio Registro AIMOVIG. URL https://www.aifa.gov.it/en/-/attiv 
azione-web-e-pubblicazione-schede-di-monitoraggio-registroaimovig (assessed 8 december 2021)

11. Russo A, Silvestro M,Scotto di Clemente F, Trojsi F, Bisecco A, Bonavita S, et al. Multidimensional assessment of the effects of erenumab in chronic migraine patients with previous unsuccessful preventive treatments: a comprehensive real-world experience. $\mathrm{J}$ Headache Pain. 2020;2020(21):69.

12. Robblee J, Devick KL, Mendez N, Potter J, Slonaker J, Starling AJ. Real-world patient experience with erenumab for the preventive treatment of migraine. Headache. 2020;2020(60):2014-25.

13. Raffaelli B, Kalantzis R, Mecklenburg J, Overeem LH, Neeb L, Gendolla A, Reuter U. Erenumab in chronic migraine patients who previously failed five first-line oral prophylactics and onabotulinumtoxina: a dual-center retrospective observational study. Front Neurol. 2020;2020(11):417.

14. Ornello R, Casalena A, Frattale I, Gabriele A, Affaitati G, Giamberardino MA, et al. Real-life data on the efficacy and safety of erenumab in the Abruzzo region, central Italy. J Headache Pain. 2020;2020(21):32.

15. Barbanti P, Aurilia C, Egeo G, Fofi L, Cevoli S, Colombo B, et al. Erenumab in the prevention of high-frequency episodic and chronic migraine: Erenumab in Real Life in Italy (EARLY), the first Italian multicenter, prospective real-life study. Headache. 2021;2021(61):363-72.

16. Vernieri F, Altamura C, Aurilia C, Brunelli N, Egeo G, Fofi L, et al. Effectiveness, safety, and tolerability of galcanezumab in a real-life setting in patients with migraine in Italy (the GARLIT study). Neurol Sci. 2020;2020(41):487-8.

17. Schiano di Cola F, Rao R, Caratozzolo S, Di Cesare M, Venturelli $\mathrm{E}$, Balducci U, et al. Erenumab efficacy in chronic migraine and medication overuse: a real-life multicentric Italian observational study. Neurol Sci 2020;41:489-90.

18. Scheffler A, Messel O, Wurthmann S, Nsaka M, Kleinschnitz C, Glas M, et al. Erenumab in highly therapy-refractory migraine patients: First German real-world evidence. J Headache Pain. 2020;2020(21):84

19. Matteo E, Favoni V, Pascazio A, Pensato U, Benini M, Asioli GM, et al. Erenumab in 159 high frequency and chronic migraine patients: real-life results from the Bologna Headache Center. Neurol Sci. 2020;2020(41):483-4.

20. Kanaan S, Hettie G, Loder E, Burch R. Real-world effectiveness and tolerability of erenumab: a retrospective cohort study. Cephalalgia. 2020;2020(40):1511-22.

21. Cheng S, Jenkins B, Limberg N, Hutton E. Erenumab in chronic migraine: an Australian experience. Headache. 2020;2020(60):2555-62.

22. Vernieri F, Altamura C, Brunelli N, Costa CM, Aurilia C, Egeo $\mathrm{G}$, et al. Galcanezumab for the prevention of high frequency episodic and chronic migraine in real life in Italy: a multicenter prospective cohort study (the GARLIT study). J Headache Pain. 2021;2021(22):35.

23. Ranieri A, Alfieri G, Napolitano M, Servillo G, Candelaresi P, Di Iorio $\mathrm{W}$, et al. One year experience with erenumab: real-life data in 30 consecutive patients. Neurol Sci. 2020;2020(41):505-6.
24. Barbanti P, Aurilia C, Cevoli S, Egeo G, Fofi L, Messina R, et al. Long-term (48 weeks) effectiveness, safety, and tolerability of erenumab in the prevention of high-frequency episodic and chronic migraine in a real world: Results of the EARLY 2 study. Headache. 2021(2021).

25. Tepper SJ, Ashina M, Reuter U, Brandes JL, Dolezil D, Silberstein $\mathrm{SD}$, et al. Long-term safety and efficacy of erenumab in patients with chronic migraine: Results from a 52-week, open-label extension study. Cephalalgia. 2020;2020(40):543-53.

26. Detke H, Pozo-Rosich P, Reuter U, Dolezil D, Li L, Wang S, Aurora S. One-year treatment with galcanezumab in patients with chronic migraine: results from the open-label phase of the REGAIN study (P2.10-010). Neurology. 2019;92:2019.

27. Dodick D. Patient perceptions and treatment preferences in migraine management. CNS Drugs. 2002;2002(16 Suppl 1):19-24.

28. Stewart WF, Lipton RB, Kolodner KB, Sawyer J, Lee C, Liberman $\mathrm{JN}$. Validity of the migraine disability assessment (MIDAS) score in comparison to a diary-based measure in a population sample of migraine sufferers. Pain. 2000;2000(88):41-52.

29. Pensato U, Baraldi C, Favoni V, Cainazzo MM, Torelli P, Querzani $\mathrm{P}$, et al. Real-life assessment of erenumab in refractory chronic migraine with medication overuse headache. Neurol Sci. 2021(2021)

30. Tepper S, Ashina M, Reuter U, Brandes JL, Dolezil D, Silberstein $S$, et al. Safety and efficacy of erenumab for preventive treatment of chronic migraine: a randomised, double-blind, placebo-controlled phase 2 trial. Lancet Neurol. 2017;2017(16):425-34.

31. Burstein R, Blumenfeld AM, Silberstein SD, Manack Adams A, Brin MF. Mechanism of action of onabotulinumtoxina in chronic migraine: a narrative review. Headache. 2020;2020(60):1259-72.

32. Blumenfeld AM, Frishberg BM, Schim JD, Iannone A, Schneider G, Yedigarova L, Manack Adams A. Real-World Evidence for Control of Chronic Migraine Patients Receiving CGRP Monoclonal Antibody Therapy Added to OnabotulinumtoxinA: A Retrospective Chart Review. Pain Ther. 2021(2021).

33. Tepper SJ, Diener HC, Ashina M, Brandes JL, Friedman DI, Reuter U, et al. Erenumab in chronic migraine with medication overuse: Subgroup analysis of a randomized trial. Neurology. 2019;2019(92):e2309-20.

34. Tepper SJ, Fang J, Vo P, Shen Y, Zhou L, Abdrabboh A, et al. Impact of erenumab on acute medication usage and health care resource utilization among migraine patients: a US claims database study. J Headache Pain. 2021;2021(22):27.

35. Silvestro M, Tessitore A, Scotto di Clemente F, Battista G, Tedeschi G, Russo A. Refractory migraine profile in CGRP-monoclonal antibodies scenario. Acta Neurol Scand. 2021(2021).

36. Deen M, Correnti E, Kamm K, Kelderman T, Papetti L, RubioBeltran E, et al. Blocking CGRP in migraine patients-a review of pros and cons. J Headache Pain. 2017;2017(18):96. 